\title{
Tricho-Dento-Osseous Syndrome: A Report of a Familial Cluster and a Literature Review
}

\author{
N. Karvelas, I. Kranias, D. Veroutis, E. A. Stylianaki, S. Sakellariou, and I. Ntanasis-Stathopoulos
}

\section{ABSTRACT}

Tricho-dento-osseous syndrome (TDO), is a very rare, autosomal dominant genetic disorder, commonly characterized by curly hair at infancy, severe enamel hypomineralization and hypoplasia with taurodontism teeth, bone defects and other deformities. Other phenotypic features include flat fingernails and altered craniofacial morphology. A genetic linkage has been identified on chromosome 17q21 in the DLX3 gene. Treatment plan of TDO is to prevent problems such as sensitivity and dental attrition of the hypoplastic structure of the tooth, to promote the esthetics and encourage self-confidence of the patient. In this case report, we present a family with the proband father, and the two children siblings affected by the TDO syndrome. We describe clinical and radiological features, along with dental characteristics and genetic background. Management of TDO syndrome necessitates a multidisciplinary approach, appropriate documentation, and long-term follow up.

Keywords: Bone, DLX3 gene, Enamel, Taurodontism, Tricho-dento-osseous syndrome (TDO).
Published Online: November 12, 2021

ISSN: 2684-4443

DOI:10.24018/ejdent.2021.2.6.110

\section{N. Karvelas}

Department of Orthodontics, School of Dentistry, Grigore T. Popa, University of Medicine and Pharmacy, Iasi, Romania.

(e-mail: karvelas93@gmail.com)

I. Kranias

Mediterraneo Dental Clinic of Athens, Athens, Greece.

(e-mail: eliassb@windowslive.com)

D. Veroutis

Laboratory of Histology-Embryology,

Molecular Carcinogenesis Group,

Medical School, National and Kapodistrian University of Athens,

Athens, Greece and Biomedical Research Foundation Academy of Athens, Athens, Greece.

(e-mail: dimitrisveroutis1@ gmail.com) E.-A. Stylianaki

Biomedical Sciences Research Centre "Alexander Fleming", Vari, Greece.

(e-mail: stylianaki@fleming.gr)

S. Sakellariou

Department of Pathology, Laiko General Hospital, Medical School, National and Kapodistrian University of Athens, Athens, Greece.

(e-mail: sakellarioustrat ${ }^{\circledR}$ yahoo.gr)

I. Ntanasis-Stathopoulos

Department of Clinical Therapeutics, School of Medicine, National and Kapodistrian University of Athens, Athens, Greece.

(e-mail: johnntanasis ${ }^{\circledR}$ med.uoa.gr)

*Corresponding Author

\section{INTRODUCTION}

Tricho-dento-osseous (TDO) syndrome is a rare, autosomal dominant genetic disorder, also known as a form of ectodermal dysplasia and first described in 1972 by Lichtenstein et al. [1]. Robinson et al. [2] had already reported a case of hereditary hypoplasia of the enamel associated with a characteristic structure of the hair without bone implication. TDO is outlined by hair defects, dental defects, bone changes, nail defects, craniofacial defects, and other reported abnormalities, and may be apparent at birth due to curly hair that is present in all affected newborns [3], [4]. More specifically, the hair defects include kinky, curly, wavy hair, the dental defects include yellow-brown teeth, severe attrition of the enamel, enamel with hypocalcification/hypomaturation, enamel hypoplasia, taurodontism in primary and permanent teeth, dental abscesses, the bone defects include sclerosis, the nail defects include splitting of the superficial area of the nails, the craniofacial defects include frontal bossing, square jaw, mandibular prognathism, maxillary retrusion, dolichocephaly. Additional abnormalities may be also present such as impacted teeth, clinodactyly and skin lesions [3], [4]. This syndrome is very rare, and a limited number of cases have been presented in the literature. Here, we present a case study of TDO syndrome with a familial link including 3 members of the same family. 


\section{CASE Presentation}

\section{A. Case 1}

A 20-year-old male patient, the son of the family was referred at our clinic for an orthodontic treatment. Panoramic and lateral cephalometry radiographs were obtained as a part for orthodontic examination. Medical, dental, and social history were collected. His past medical history was unremarkable. The patient presented yellowish-brown teeth and the panoramic radiography showed severe enamel attrition on the central incisors, lateral incisors, and canines both in the maxilla and mandible arch (Fig. 1). The intraoral examination of the patient indicated hypoplastic teeth, lack of the enamel and dentin exposure to the oral environment, as sequence of the dentin friction (Fig. 1). Also, crowding and an ectopic canine were noted. Blood tests were within normal ranges. Extraoral characteristics as bones, hair and nails were normal (Fig. 1C).

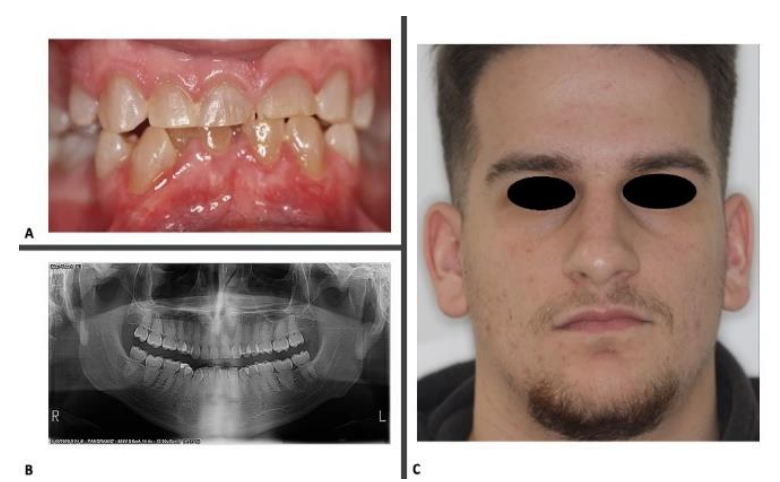

Fig. 1. A) Intraoral view of the son demonstrates yellowish-brown teeth, enamel attrition and dentin exposure. B) Panoramic view of the son shows severe enamel attrition on the anterior segment. C) The son expresses normal extraoral characteristics.

\section{B. Case 2}

The 22-year-old sister of the family expressed identical dental clinical findings. Intraoral examination demonstrated yellowish-brown teeth, severe discoloration and enamel attrition (Fig. 2). She complained about increased tendency of teeth fracture. As a result, she had already two dental implants due to loss of first permanent lower molars. Her bones, hairs and nails were normal.

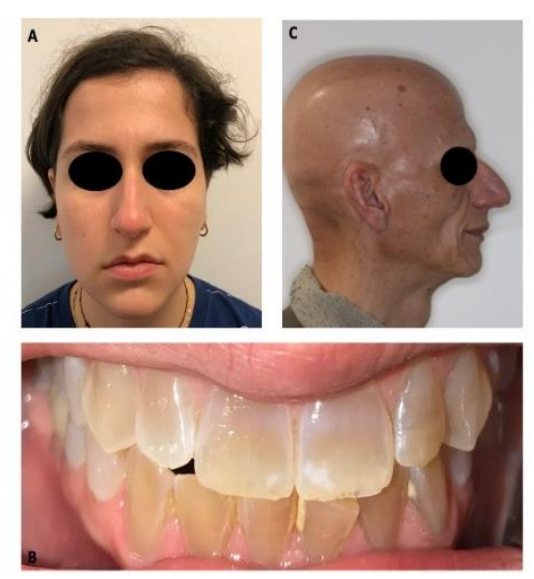

Fig. 2. A-B) The sister presents normal extraoral characteristics and yellowish-brown teeth with enamel attrition intraorally. C) The proband demonstrate total lack of hair (alopecia).

\section{Case 3}

The 56-year-old father was subsequently involved in our clinical evaluation. The clinical examination revealed alopecia, flat fingernails and microsomia (Fig. 2 C). The patient was diagnosed with completely edentulous (totally anodontia) having lost all his teeth for 20 years ago, reporting exactly the same hypoplastic teeth with enamel attrition and the same discoloration similar with his children. The patient reported that the nails have a tendency to break or peel.

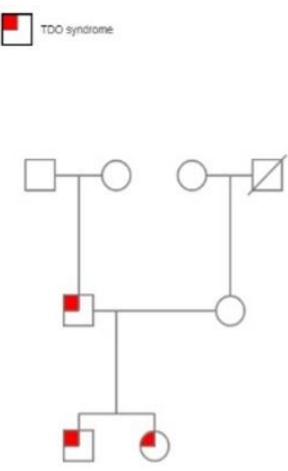

Fig. 3. Pedigree of the fam illy with TDO syndrome.

A pedigree family was designed for genetic evaluation. The father, the son, and the sister of the family present with clinical characteristics highly suggestive of TDO syndrome. The mother has normal hair without any dental defects on the remaining teeth. The pedigree family exhibits that grandparents of the proband including his mother indicate normal characteristics (Fig. 3). Interrogating the family, we affirm that the father is the proband who first presented and expressed the clinical characteristics of the genetic disease. He presented "de novo" (anew) genotype and phenotype mutation. However, the genetic analysis was performed in the coding region of the gene DLX3 by polymerase chain reaction (PCR). There is no detection of potentially pathogenic variant or pathogenic mutation in the coding region of the gene DLX3.

\section{DISCUSSION}

The challenging diagnosis of TDO in dentistry is determined by the appropriate recognition of the severe hypoplasia of the enamel, as well as the need for differentiation from amelogenesis imperfecta with taurodontism. This can be especially challenging based on clinical and radiographic criteria exclusively [14]. Therefore, further histologic, and genetic investigations are required for establishing the diagnosis and formulating the optimal management of the patients.

As far as the genetic background is concerned, the TDO is transmitted as a highly penetrant autosomal dominant trait [12]. The syndrome is depicted to a locus on chromosome 17q21, where the distal-less homeobox gene incorporate the DLX3 and DLX7 gene respectively. Molecular scanning displays a 4 bp (base-pair) deletion mutation of DLX3 (c.571_574delGGGG). This deletion creates a frameshift mutation and, as a result, a truncated protein is unable to form a dimer and bind to the promoter of enamel matrix protein genes (Amelx, Enam, Klk4, and Odam) [11], [13]-[15].

DLX3 (Distal-Less Homeobox 3) is a protein coding gene 
which is expressed both during embryonic development in neural crest cells and the placenta, as well as in differentiated tissues such as hair matrix cells, dental epithelium, odontoblast. The levels of DLX3 gene expression are contextdependent and vary among terminally differentiated ameloblasts, odontoblasts, osteoblasts, chondroblasts and chondrocytes. It functions, thus, as a key-regulator of osteogenesis, mainly through $\mathrm{Wnt} / \beta$-catenin signaling. In addition to this, DLX3 mutations result in an increase of the mineral density of the bone that in turn leads to endochondral ossification [10], [11], [14], [16]-[18]. Interestingly, the characteristic phenotype of TDO syndrome affecting teeth, hair and bone coincides with the tissues expressing DLX3. Furthermore, Price et al. described that TDO has a greater phenotypic heterogeneity and that is the result of both environmental factors and other genetic modifiers [8], [11].

Wright et al. discovered a new mutation in the DLX3 gene with the same two base pair deletion (c561_562delCT) where all affected subjects had reduced enamel thickness and less severe taurodontism compared with the 4 bp deletion mutation. Also, DEXA evaluations indicated a slightly increased bone density but without exposing sclerotic bone defects. The authors concluded that this new mutation represents an impaired phenotype of TDO [16]. According to Shapiro et al. TDO has three subtypes, TDO I, TDO II and TDO III, that share common characteristics and are differentiated by specific variations in dental presentation [4].

Regarding the dental defects in TDO syndrome, the primary and permanent teeth are yellowish-brown in color and exhibit a generalized attrition, displaying as enamel calcification defects or enamel hypomaturation defects together with enamel hypoplasia [1], [2], [7], [14]. The enamel is thin and defined to be approximately $1 / 4$ to $1 / 8$ of the thickness of the normal enamel. In the $90 \%$ of the cases, the pulp chamber is very wide and pulp horns extend to the enamel-dentin junction, which often results in pulp necrosis and periapical abscesses [7], [9], [14]. Moreover, a partial agenesis is observed in the TDO syndrome, along with late eruption and taurodontism of the molars, which is a developmental condition where the body of the tooth is elongated at the expense of the root leading to large pulp chamber [20]. TDO dental findings are similar to amelogenesis imperfecta, an enamel anomaly which is a genetically distinct condition as defined and described by Prince et al. [19].

In addition to the above, TDO is associated with bone cortical sclerosis, most frequently recorded at the base of the skull, the mastoids, and the transitional zones in the long bones; a condition predisposing for bone fractures [14]. Nevertheless, bone density and thickness are not considered as pathognomonic criteria of TDO syndrome [12]. Also, kinky, curly, and wavy hair have been described as hair defects and may be a unique feature differentiating TDO from the hypomaturation-type of amelogenesis imperfecta [3], [5]. Nevertheless, it has to be noted that $55 \%$ of TDO individuals have straighten hair throughout childhood [21]. In addition, splitting of the superficial area of the nails and toenails can be suggestive of TDO. Other deficiencies can be impacted teeth, clinodactyly and skin lesions. Craniofacial deformities can develop as frontal bossing, square jaw, mandibular prognathism, maxillary retrusion and a dolichocephalic type of face [5]. Furthermore, the most updated study including the largest sample of patients confirmed that the position of the maxilla is more retrusive in comparison with the cranial base in TDO affected individuals [14].

\section{CONCLUSIONS}

The clinical and radiographical manifestations observed in the two siblings and the father correspond well to a familial cluster of the TDO syndrome. Management of TDO syndrome necessitates a multidisciplinary approach, appropriate documentation, and long-term follow up. A more in-depth molecular analysis might reveal the underlying genetic spectrum of this complex and multifaceted disease.

\section{ETHICS}

No ethics approval was required for this study.

\section{FUNDING}

None.

\section{CONFLICTS OF INTEREST}

The authors declare no relevant conflicts of interest.

\section{INFORMED CONSENT}

Informed consent was received and approved by all members of the family.

\section{REFERENCES}

[1] Lichtenstein J, Warson R, Jorgenson R, Dorst JP, McKusick VA. The tricho-dento-osseous (TDO) syndrome. American Journal of Human Genetics. 1972;24(5):569-582.

[2] Robinson GC, Miller JR, Worth HM. Hereditary enamel hypoplasia: its association with characteristic hair structure. Pediatrics. 1966;37(3):498-502.

[3] Witkop CJ, Worth HM. Tricho-dento-osseous syndrome. Birth defects compendium: Bergsma D. National Foundation March of Dimes, second edition. 1973.

[4] Al-Batayneh OB. Tricho-dento-osseous syndrome: Diagnosis and dental management. International Journal of Dentistry. 2012:1-9.

[5] Lichtenstein J, Warson RW. Syndrome of dental anomalies, curly hair and sclerotic bones. Birth Defects Original Article Series. 1971;7(7):308-311.

[6] Shapiro SD, Quattromani FL, Jorgenson RJ, Young RS. Tricho-dentoosseous syndrome: heterogeneity or clinical variability. American Journal of Medical genetics. 1983;16(2):225-236.

[7] Quattromani F, Shapiro SD, Young RS, et al. Clinical heterogeneity in the trichodento-osseous syndrome. Human Genetics. 1983:64(2):116121.

[8] Price JA, Wright JT, Kula K, Bowden DW, Hart TC. A common DLX3 gene mutation is responsible for tricho-dento-osseous syndrome in Virginia and North Carolina families. Journal Medical genetics. 1988; 35:825-828.

[9] Parul J, Rahul K, Subrata S, Subir S. Tricho-dento-osseous syndrome and precocious eruption. Journal of clinical and experimental dentistry. 2017;9(3): e494-e497.

[10] Robinson GW, Mahon KA. Differential and overlapping expression domains of Dlx-2 and Dlx-3 suggest distinct roles for Distal-less homeobox genes in craniofacial development. Mechanisms of Development. 1994;48(3):199-215.

[11] Price JA, Bowden DW, Wright JT, Pettenati MJ, Hart TC. Identification of a mutation DLX3 associated with tricho-dentoosseous (TDO) syndrome. Human Molecular Genetics. 1998;7(3)563569. 
[12] Hart TC, Bowden DW, Bolyard J, Kula K, Hall K, Wright JT. Genetic linkage of the tricho-dento-osseous syndrome to chromosome $17 \mathrm{q} 21$. Human Molecular Genetics. 1997;6(13):2279-2284.

[13] Choi SJ, Song IS, Ryu OH, et al. A 4bp deletion mutation in DLX3 enhances osteoblastic differentiation and bone formation in vitro. Bone. 2008;42(1):162-171.

[14] NGutenberg T, Phillips C, Frazier-Bower S, Wright T. Craniofacial variations in the tricho-dento-osseous syndrome. Clin Genet. 2013;83(4):375-9.

[15] Zhang Z, Tian H, Lv P, Wang W, Jia Z, Wang S, Zhou C, Gao X. Transcriptional factor DLX3 promotes the gene expression of enamel matrix proteins during amelogenesis. PLoS One. 2015;10(3):e0121288.

[16] Wright JT, Hong SP, Simmons D, Daly B, Uebelhart D, Lunder HU. DLX3 c.561_562delCT mutation causes attentuated phenotype of tricho-dento-osseous syndrome. Am $J$ Med Genet A. 2008;146A(3):343-9.

[17] Ghoul-Mazgar S, Hotton D, Lezot F, Blin-Wakkach C, Asselin A, Sautier JM, Bernal A. Expression pattern of Dlx3 during cell differentiation in mineralized tissues. Bone. 2005;37(6):799-809.

[18] Viale-Bouroncle S, Klingelhoffer C, Ettl T, Reichert TE, Morsczeck C. A protein kinase A (PKA)/ $\beta$-catenin pathway sustains the BMP2/DLX3-induced osteogenic differentiation in dental follicle cells (DFCs). Cell Signal. 2015; 27(3):598-605.

[19] Price JA, Wright JT, Walker SJ, Crawford PJ, Aldried MJ, Hart TC. Tricho-dento-osseous syndrome and amelogenesis imperfecta with taurdontism are genetically distinct conditions. Clin Genet 1999;56:35-40.

[20] Dong J, Amor D, Aldred MJ, Gu T, Escamilla M, MacDougall M. DLX3 mutation associated with autosomal dominant amelogenesis imperfecta with taurodontism. Am J Med Genet A. 2005;133:138-41. 